\title{
Galactic AGB stars from the IPHAS survey
}

\author{
N. J. Wright, ${ }^{1}$ M. J. Barlow ${ }^{2}$ R. Greimel,${ }^{3}$ J. E. Drew ${ }^{4}$ and \\ M. Matsuura ${ }^{2}$ \\ ${ }^{1}$ Harvard-Smithsonian Center for Astrophysics, 60 Garden Street, Cambridge, MA 02138 \\ ${ }^{2}$ University College London, Gower Street, London WC1E 6BT, U.K. \\ ${ }^{3}$ Institut für Physik, Karl-Franzen Universität Graz, Universitätsplatz 5, 8010 Graz, Austria \\ ${ }^{4}$ University of Hertfordshire, College Lane, Hatfield, AL10 9AB, U.K.
}

\begin{abstract}
We present a photometric analysis of the properties of asymptotic giant branch stars identified in the INT Photometric H-alpha Survey (IPHAS) of the northern Galactic plane. Follow-up spectroscopy has revealed that the IPHAS ( $\mathrm{r}-\mathrm{Ha}$ ) colour is a valuable diagnostic of the photospheric $\mathrm{C} / \mathrm{O}$ ratio, and may be used to identify hundreds of carbon and S-type stars.
\end{abstract}

Keywords. stars: AGB and post-AGB - stars: carbon - infrared: stars - techniques: spectroscopic

Asymptotic giant branch (AGB) stars are one of the final evolutionary stages of all intermediate mass stars and one of the major sources of enriched material returned to the interstellar medium, including all s-process elements and the majority of carbon. The INT Photometric H $\alpha$ Survey (IPHAS, Drew et al. 2005) is imaging the entire northern Galactic plane using Sloan $r^{\prime}, i^{\prime}$, and narrow-band $\mathrm{H} \alpha$ filters. One of the unique features of the IPHAS colour-colour diagram is that the main-sequence and giant branches are well separated at late spectral types, effectively allowing the AGB population across the entire northern Galactic plane to be identified and studied (Wright et al. 2008)

Follow-up spectroscopy on a number of optical and near-IR instruments has allowed this population to be studied in more detail. Wright et al. (2009) published a near-IR spectral library of AGB stars with a particular focus on very late-type sources. The spectral library includes spectra in all three near-IR bands as well as many variable objects and chemically evolved sources such as S-type and carbon stars. The library includes spectral classification sequences highlighting the various molecular features identified and discusses a number of rare features and the potential molecules responsible.

Finally we discuss a correlation between the IPHAS $\left(r^{\prime}-\mathrm{H} \alpha\right)$ colour and the C/O abundance index (Keenan \& Boeshaar, 1980). Wright et al. (2009) found that the IPHAS $\left(r^{\prime}-\mathrm{H} \alpha\right)$ colour could be used to estimate $\mathrm{C} / \mathrm{O}$ ratios for S-type stars and therefore determine their state of chemical evolution in the transition from O-rich $(\mathrm{C} / \mathrm{O}<1)$ to carbon rich $(\mathrm{C} / \mathrm{O}>1)$ via the intermediate S-type phase $(\mathrm{C} / \mathrm{O} \sim 1)$. When combined with a near-IR colour the relationship has the potential to separate O-rich, S-type, and carbon stars across the Galactic plane based on their photometry alone, which will increase our understanding of AGB dredge-up mechanisms, galactic chemical evolution, and the structure and metallicity of the Galactic disk.

\section{References}

Drew, J. E., Greimel, R., Irwin, M. J. et al. 2005, MNRAS, 362, 753

Keenan, P. C. \& Boeshaar, P. C. 1980 ApJS, 43, 379

Wright, N. J., Greimel, R., Barlow, M. J. et al. 2008, MNRAS, 390, 929

Wright, N. J., Barlow, M. J., Greimel, R. et al. 2009, MNRAS, in press 